\title{
THE VELOCITY DISTRIBUTION OF HYPERVELOCITY STARS
}

\author{
Elena M. Rossi ${ }^{1,2}$, Shiho Kobayashi ${ }^{3,2}$, and Re'em SARI ${ }^{2,4}$ \\ ${ }^{1}$ Leiden Observatory, Leiden University, P.O. Box 9513, 2300 RA Leiden, The Netherlands \\ ${ }^{2}$ Racah Institute of Physics, Hebrew University, Jerusalem, 91904, Israel \\ 3 Astrophysics Research Institute, Liverpool John Moores University, UK \\ ${ }^{4}$ Theoretical Astrophysics 350-17, California Institute of Technology, Pasadena, CA 91125, USA \\ Received 2013 July 3; accepted 2014 July 11; published 2014 October 22
}

\begin{abstract}
We consider the process of stellar binaries tidally disrupted by a supermassive black hole (BH). For highly eccentric orbits, as one star is ejected from the three-body system, the companion remains bound to the BH. Hypervelocity stars (HVSs) observed in the Galactic halo and S-stars observed orbiting the central BH may originate from such mechanism. In this paper, we predict the velocity distribution of the ejected stars of a given mass, after they have traveled out of the Galactic potential. We use both analytical methods and Monte Carlo simulations. We find that each part of the velocity distribution encodes different information. At low velocities $<800 \mathrm{~km} \mathrm{~s}^{-1}$, the Galactic potential universally shapes the observed distribution, which rises toward a peak, related to the Galactic escape velocity. Beyond the peak, the velocity distribution depends on binary mass and separation distributions. Finally, the finite star life introduces a break related to their mass. A qualitative comparison of our models with current observations shows the great potential of HVSs to constrain bulge and Galactic properties. Standard choices for parameter distributions predict velocities below and above $\sim 800 \mathrm{~km} \mathrm{~s}^{-1}$ with equal probability, while none are observed beyond $\sim 700 \mathrm{~km} \mathrm{~s}^{-1}$ and the current detections are more clustered at low velocities $300-400 \mathrm{~km} \mathrm{~s}^{-1}$. These features may indicate that the separation distribution of binaries that reach the tidal sphere is not flat in logarithmic space, as observed in more local massive binaries, but has more power toward larger separations, enhancing smaller velocities. In addition, the binary formation/evolution process or the injection mechanism might also induce a cut-off $a_{\min } \sim 10 R_{\odot}$ in the separation distribution.
\end{abstract}

Key words: binaries: general - Galaxy: center - Galaxy: halo - Galaxy: kinematics and dynamics - Galaxy: stellar content

Online-only material: color figures

\section{INTRODUCTION}

In the last decade, an increasing number of stars were detected traveling away from our Galactic center with velocities that exceed the escape velocity from the Galaxy (Brown et al. 2005, 2007, 2009b, 2012b; Hirsch et al. 2005). These stars seem to be consistent with a Galactic center origin (see, however, Edelmann et al. 2005, but also Brown et al. 2010). These stars, traveling with radial velocities over $\sim 300 \mathrm{~km} \mathrm{~s}^{-1}$ in the halo, are called "hypervelocity stars" (HVSs). Until recently, the discovery technique automatically selected massive stars, mainly B stars with $M \sim 3-4 M_{\odot}$ (e.g., Brown et al. 2007). In the last two years, there has been an observational effort to extend the search to an older population (A-stars) of HVS (Brown et al. 2009a; Kollmeier et al. 2009, 2010), but none have yet been found.

HVSs are observed in the Galactic halo, but they seem to originate from the Galactic center. Observations of the innermost region $(<0.5 \mathrm{pc})$ of our Galactic center have revealed young stars in highly eccentric orbits. Between 0.04 and $0.5 \mathrm{pc}$, the stars form one, maybe two, disk-like structures (Levin \& Beloborodov 2003; Lu et al. 2009). At smaller distances, $<0.01 \mathrm{pc}$, instead, they are distributed in a more isotropic fashion. These latter are called S-stars and their orbits are the strongest observational evidence of the presence of a central black hole (BH; e.g., Genzel et. al. 1996; Ghez et al. 1998) with mass $M \approx 4 \times 10^{6} M_{\odot}$ (e.g., Ghez et al. 2008).

Here there is the intriguing possibility that the origin of these stars, and in particular of the S-stars, is related to that of HVSs (e.g., Perets et al. 2007). Theoretically, it has been known for a long time that a tidal field of a central $\mathrm{BH}$ can break apart the occasional star-binary entering its tidal sphere of influence, capturing one star and ejecting the other (Hills 1988). The velocity with which a star leaves the BH potential well-the ejection velocity -is of the order of $v \approx v_{\mathrm{b}}\left(M / m_{\mathrm{t}}\right)^{1 / 6}$, where $v_{\mathrm{b}}$ is the binary orbital velocity, and $m_{\mathrm{t}}$ is its total mass (Yu \& Tremaine 2003). Such a velocity can indeed be enough $\left(\geqslant 1000 \mathrm{~km} \mathrm{~s}^{-1}\right)$ to climb out of the Galactic potential and produce a star running through the Galactic halo with hundreds of $\mathrm{km} \mathrm{s}^{-1}$, as observed. Correspondingly, the semi-major axis of the captured star $a_{\mathrm{c}} \approx G M /(-2 \epsilon) \leqslant 0.02 \mathrm{pc}$, where the specific energy is $|\epsilon|=v^{2} / 2$.

After the discovery of the first HVS, there have been considerable theoretical efforts to determine in more detail the consequence of binary tidal disruption (e.g., Gualandris et al. 2005; Ginsburg \& Loeb 2006; Bromley et al. 2006; Sesana et al. 2007; Perets et al. 2007; Kenyon et al. 2008; Lu et al. 2010; Sari et al. 2010; Antonini et al. 2010; Zhang et al. 2010, 2013; Kobayashi et al. 2012). In Sari et al. (2010, , hereafter SKR) and Kobayashi et al. (2012), we proposed a new semi-analytic treatment of the three-body interaction, exploiting the large disparity in mass $\left(M / m_{\mathrm{t}}\right) \gg 1$. This method allows us to describe a binary-BH encounter independently of most physical properties of the binary, such as its semi-major axis and the stars' masses. In particular, we calculated the probability of a binary surviving disruption and, when disruption occurs, the final energies of both stars (which are equal but in sign). Indeed, we showed that the integration of the binary orbit gives, for the final energy, just a numerical factor that multiplies the analytic expectation (see Section 2). In addition, we found that-for a parabolic 
trajectory of the binary center of mass - the ejection probability is independent of the mass of the star, yet the heavier component of the binary, if ejected, would escape at a smaller velocity. Notably, we also showed that the ejection velocity is largely independent of how deep the binary plunges in the sphere of influence. In particular, the ejection velocity does not increase for deeper encounters, as commonly assumed. Finally, we showed in Kobayashi et al. (2012) that a parabolic orbit well describes the orbit of stellar binaries coming from the edge of the sphere of influence of $\mathrm{SgA}^{*}$.

In this paper, we assume a statistical description of the incoming binaries and calculate the velocity distribution of the ejected stars, using our restricted three-body method and its results. This method has never been used before for such a goal, which previously relied on full three-body calculations. The main advantage of our method is that it is computationally inexpensive as well as accurate, and the main features of its results can be reproduced analytically. It therefore allows us to investigate different mass and semi-major axis distributions for the incoming binaries in a wide range of values. Moreover, it makes it particularly easy to pin down which type of binaries contribute most to a given range of ejection velocities.

Exploiting these features of the method, the calculations in this paper are first performed analytically. We assume a simplified picture of the binary-BH encounter and identify which binaries (mass ratio and separation) dominate the production of an HVS with a given velocity and mass. These results are then checked against Monte Carlo calculations. The latter use full numerical integration of binary orbits, and provide a detailed description of the velocity distributions.

Qualitatively, tidal disruptions of binaries can yield HVSs: indeed, the maximum possible velocity predicted by SKR $\left(v_{\max } \approx 5000-7000 \mathrm{~km} \mathrm{~s}^{-1}\right)$ substantially exceeds that seen observationally. A detailed prediction of the velocity distribution and a comparison with current data is therefore required in order to ascertain whether the observed distribution is quantitatively consistent with the predictions of a star binary disruption model. This is the goal of this paper. Toward this aim, our findings may be used to better plan future observational campaigns.

The paper is organized as follows. In Section 2, we describe the model we assume for the binary-BH encounter. In Section 3, we describe our assumptions for the distribution of the binary properties (masses, semi-major axis, closest approach). A simplified version of our findings in SKR allows us to analytically derive the velocity distributions of HVS (Section 4). Our analytical findings are validated against proper orbit integrations in Section 6, where we also perform a comparison with the current sample of unbound HVSs. We discuss our results and conclude in Section 7.

\section{BINARIES DISSOLVED BY BLACK-HOLE TIDES}

We consider a star with mass $m_{*}$ ejected from a binary system with semi-major axis $a$ and companion mass $m_{\mathrm{c}}$, after a close encounter with a BH of mass $M \gg m_{\mathrm{t}}$, where $m_{\mathrm{t}}=m_{\mathrm{c}}+m_{*}$.

We define the penetration factor $D=r_{\mathrm{p}} / r_{\mathrm{t}}$, which measures how deeply into the tidal sphere of radius

$$
r_{\mathrm{t}}=a\left(\frac{M}{m_{\mathrm{t}}}\right)^{1 / 3}
$$

a binary with periapsis $r_{\mathrm{p}}$ penetrates. The tidal radius is defined as the distance from the $\mathrm{BH}$ where the mutual gravity of the binary equals the $\mathrm{BH}$ tidal pull. When the binary approaches the
$\mathrm{BH}$, the orbit of the binary's center of mass has a semi-major axis $r_{\mathrm{a}}$ comparable to the $\mathrm{BH}$ sphere of influence $\sim$ a few parsecs or $r_{\mathrm{a}}>10^{3} r_{\mathrm{t}}$, for $a \lesssim 0.1 \mathrm{AU}$. The orbital eccentricity is thus $1-e=D\left(r_{\mathrm{t}} / r_{\mathrm{a}}\right)<10^{-3}$, and the orbit can be described with a parabola. In this case, which star is ejected after disruption does not depend on its mass, but on its relative position with respect to the $\mathrm{BH}$ at the tidal radius (measured in terms of an angle called "the binary phase"). Not all binaries, however, are disrupted as a result of the gravitational encounter. For planar and circular binaries, we showed that the disruption probability, averaged over the binary phase and sense of rotation, is a non-monotonic function of $D$ that does not saturate to 1 for $D \rightarrow 0$. Rather, $\sim 20 \%$ of the binaries survive disruption for $D \ll 1$ (see Figure 4 in SKR). In this paper, we allow for different inclinations and we obtain that a smaller percentage, about $\sim 10 \%$, survives for $D \rightarrow 0$ (S. Kobayashi et al., in preparation).

When the binary dissolves, the ejection velocity is

$$
v \cong \sqrt{\frac{2 G m_{\mathrm{c}}}{a}}\left(\frac{M}{m_{\mathrm{t}}}\right)^{1 / 6} .
$$

This is formally the velocity at infinity, solely in the presence of the $\mathrm{BH}$ potential. Practically, the star velocity may be considered constant and equal to Equation (2) at a finite distance of $\approx 100 r_{\mathrm{t}}$, which, for binary separation $a \leqslant 60 \mathrm{AU}$, is smaller than the sphere of influence of the $\mathrm{BH}(\approx 3 \mathrm{pc}$; Schödel et al. 2003). This range of separations includes all the tight binaries that indeed will produce HVSs. Beyond a few parsecs, deceleration from the Galactic potential should be taken into account (Kenyon et al. 2008). In Equation (2), there is a numerical coefficient missing, which depends on the binary phase, its penetration factor, the binary eccentricity, and the inclination of its orbit. However, the mean velocity over the binary phase is almost independent of the penetration factor and the coefficient is very close to unity (see Figure 10 in SKR).

For our analytical calculations, we thus assume a simple description of the disruption process where only and all binaries approaching the $\mathrm{BH}$ with $D \leqslant 1$ will be tidally disrupted and the final specific energy after breakup is $\pm \epsilon=v^{2} / 2$, where $v$ is given by Equation (2). In the Monte Carlo simulations presented later, instead, we numerically compute the final energy for circular binaries, if the binary actually gets disrupted by the encounter with the BH. In the Monte Carlo approach, we thus exclude the two main assumptions of our analytical method: the use of Equation (2) with a unity proportional factor and of a step function probability distribution, where survival occurs only for $D>1$. We will show that our analytical predictions are overall validated, but there are qualitative differences in the fastest branches of the velocity distribution, when using a more precise calculation of the disruption energy (Section 6).

\section{BINARY STATISTICAL DESCRIPTION}

The post-disruption distributions of ejection velocities depend on the physical properties of the binaries that reach the $\mathrm{BH}$ within its tidal radius $\left(r_{\mathrm{p}}<r_{\mathrm{t}}\right)$. These are binaries with specific orbital angular momentum $J$ around the $\mathrm{BH}$ smaller than $J_{\mathrm{lc}} \simeq \sqrt{2 G M r_{\mathrm{t}}}$. These orbits are called "loss-cone" orbits. The way stars enter the loss cone depends on the relaxation processes that redistribute stars in phase space (Lightman \& Shapiro 1977). In this paper we do not thoroughly investigate the rate of production of HVS. We cite two extreme limits.

If the rms change $\Delta J$ in angular momentum over an orbital period is small, $\Delta J \ll J_{\mathrm{lc}}$, the binary diffuses in phase space 
until $J \simeq J_{\text {lc }}$ and the binary center of mass approaches the $\mathrm{BH}$ with a flat distribution in $D$. In this regime, the rate of disruption of binaries is about one star every orbit at the radius of influence of the BH. Somewhat counterintuitively, this rate is independent of the binaries' tidal disruption radius. We call this regime the "empty loss-cone regime."

In the opposite case of a "full loss-cone regime," the change in angular momentum over an orbital period is $\Delta J \gg J_{\text {lc }}$. Then the loss cone is continuously and uniformly refilled. In this case, the fraction of stars with apocenter $r_{\mathrm{a}}$, but with periapsis distance $r_{\mathrm{p}} \ll r_{\mathrm{a}}$, is of the order of $r_{\mathrm{p}} / r_{\mathrm{a}}$. The rate of production of HVS is therefore proportional to the tidal radius. Since $r_{\mathrm{t}} \propto a$, wider binaries are more frequently disrupted.

Observationally, massive binaries have a uniform distribution in the logarithm of the period (Duquennoy \& Mayor 1991; Kiminki \& Kobulnicky 2012), which translates into a semimajor axis distribution

$$
f_{\mathrm{a}} \propto \frac{1}{a}
$$

where the minimum separation $a_{\text {min }}$ should be chosen so to exclude binaries that are close enough that they can undergo mass transfer between the binary members (Eggleton 1983). We will discuss the conditions in Section 4.1 when the highest velocities are evaluated. The upper limit is not very relevant to this investigation as wide binaries produce very low ejection velocities. There is observational and theoretical evidence that massive binaries host approximately equal-mass stars (Kobulnicky \& Fryer 2007; Krumholz et al. 2009; Kiminki \& Kobulnicky 2012). However, selection effects make it difficult to detect binaries with significantly different mass components, and a full observational determination of the distribution of binary masses is not available. Here we will consider two extreme cases: (1) all binaries are composed of equal-mass stars, or (2) the companion mass is drawn from a power-law mass function,

$$
f_{\mathrm{m}} \propto m_{\mathrm{c}}^{-\alpha}
$$

for $m_{\min } \leqslant m_{\mathrm{c}} \leqslant m_{\max }$. We define $R_{\min }$ and $R_{\max }$ the radii of stars with mass $m_{\min }$ and $m_{\max }$, respectively. A Salpeter mass function has $\alpha=2.35$. For numerical evaluations, we assume hereafter a low-mass cut-off of $m_{\min }=0.5 M_{\odot}$ and a high-mass cut-off of $m_{\max }=100 M_{\odot}$. In our calculations, we will explore the dependence on the index $\alpha$, but we will confine ourselves to $\alpha>1$, which corresponds to most stars being of low mass, as normally observed.

Finally, we will adopt a linear relation between the radius of the star and its mass: $R_{\mathrm{c}} \propto m_{\mathrm{c}}$. This follows from hydrostatic equilibrium and the approximate constancy of the central temperature for main sequence star. More accurate treatments show that $R_{\mathrm{c}} \propto m_{\mathrm{c}}^{0.8}$, which would not change our conclusions.

\section{VELOCITY DISTRIBUTION: ANALYTICAL METHOD}

If a star with given mass $m_{*}$ is ejected as a consequence of a binary tidal disruption by a $\mathrm{BH}$ of mass $M$, what is the probability distribution of its ejection velocity $v$ ?

For given masses of the HVS and the $\mathrm{BH}$, the ejection velocity is a function of the binary semi-major axis and the companion mass: $v=v\left(a, m_{\mathrm{c}}\right)$ as given by Equation (2),

$$
v \propto \begin{cases}a^{-1 / 2} m_{\mathrm{c}}^{1 / 2}, & m_{\mathrm{c}} \ll m_{*}, \\ a^{-1 / 2} m_{\mathrm{c}}^{1 / 3}, & m_{\mathrm{c}} \gg m_{*} .\end{cases}
$$

We denote by $\dot{N}_{\mathrm{v}}$ the number of stars with velocities of the order of $v$ ejected from the vicinity of the massive BH per unit time. Given a velocity distribution $f_{\mathrm{v}}$, the rate is $\dot{N}_{\mathrm{v}} \propto v \times f_{\mathrm{v}}$. In this paper we do not calculate the normalization of $\dot{N}_{\mathrm{v}}$, but instead focus on its functional shape as a function of $v$ (i.e., $f_{\mathrm{v}}$ ). In principle, $\dot{N}_{\mathrm{v}}$ should be obtained by integrating over the contribution of all binaries. However, the main features of this function can be obtained analytically as follows. We first calculate how the probability density behaves, fixing one parameter at a time: the binary semi-major axis or the companion mass. Then, we focus on the binaries that are responsible for the highest ejection velocities and calculate their distribution. From these results, it is possible to infer which binaries contribute most to a given ejection velocity $v$. Illustrations will visualize the method, complementing the explanation in the text.

The rate, $\dot{N}_{\mathrm{v}}$, at which stars are ejected from the Galactic $\mathrm{BH}$ with velocity of the order of $v$ is not, however, the quantity that we need to compare with observations. In fact, we observe HVSs in the Galactic halo after they have traveled far from their birth place. Additional effects, specifically the Galactic potential and the finite age of the stars, should also be taken into account. We leave that for Section 5 .

\subsection{The Empty Loss-cone Regime}

When diffusion is the process that determines how binaries fall into the loss cone, the binary approaching rate is almost independent of the size of the loss cone itself (Lightman \& Shapiro 1977).

If we fix $a$, a mass interval between $m_{\mathrm{c}}$ and $m_{\mathrm{c}}+d m_{\mathrm{c}}$ corresponds to a given velocity interval between $v$ and $v+d v$. Since the number of ejected stars in these two ranges should be the same, the probability density for a fixed semi-major axis $a,\left.f_{\mathrm{v}}(v)\right|_{\mathrm{a}}$, is such that $\left.f_{\mathrm{v}}(v)\right|_{\mathrm{a}} d v=f_{\mathrm{m}} d m_{\mathrm{c}}$. The relative number of ejected stars with velocity $v$ for a fixed $a$ is then $v \times\left. f_{\mathrm{v}}(v)\right|_{\mathrm{a}} \propto m_{\mathrm{c}}^{-(\alpha-1)}$. Therefore,

$$
v \times\left. f_{\mathrm{v}}(v)\right|_{\mathrm{a}} \propto \begin{cases}v^{-2(\alpha-1)}, & m_{\mathrm{c}} \ll m_{*}, \\ v^{-3(\alpha-1)}, & m_{\mathrm{c}} \gg m_{*},\end{cases}
$$

where here and in the following $\left.X\right|_{\mathrm{y}}$ indicates a quantity $\mathrm{X}$ calculated at fix $Y$ and we used Equation (5). In Figure 1, Equation (6) is plotted with thin solid lines for decreasing separation $a$ toward the right. The break occurs at the ejection velocity that corresponds to an equal-mass binary $\left(m_{\mathrm{c}}=m_{*}\right)$, which decreases with $a$ as $a^{-1 / 2}$ (Equation (5)). The probability density associated with this velocity is simply the probability density for a fixed companion mass, $\left.f_{\mathrm{v}}(v)\right|_{m_{\mathrm{c}}}$, evaluated at $m_{\mathrm{c}}=m_{*}$. Since $\left.f_{\mathrm{v}}(v)\right|_{m_{\mathrm{c}}} d v=f_{\mathrm{a}} d a$, we get

$$
v \times\left. f_{\mathrm{v}}(v)\right|_{m_{\mathrm{c}}}=a \times f_{\mathrm{a}}=\text { constant, }
$$

which is constant for our standard choice of $f_{\mathrm{a}}$ (Equation (3)).

We now specifically consider binaries that produce the fastest ejections. The highest velocities are attained for small binary separations and large masses. However, the sum of the stellar radii-which are proportional to their masses-cannot exceed $a$. In our analytical treatment, unless otherwise stated, we will ask a simple relation $a>R_{\mathrm{c}}+R_{*}$. This implies that for a fixed, small $a$, the maximum companion mass could be smaller than $m_{\max }$ and therefore the maximum velocity could be less than $v\left(a, m_{\max }\right)$.

A more realistic model requires a larger minimum separation. Stars with separations less than a few stellar radii would fill 


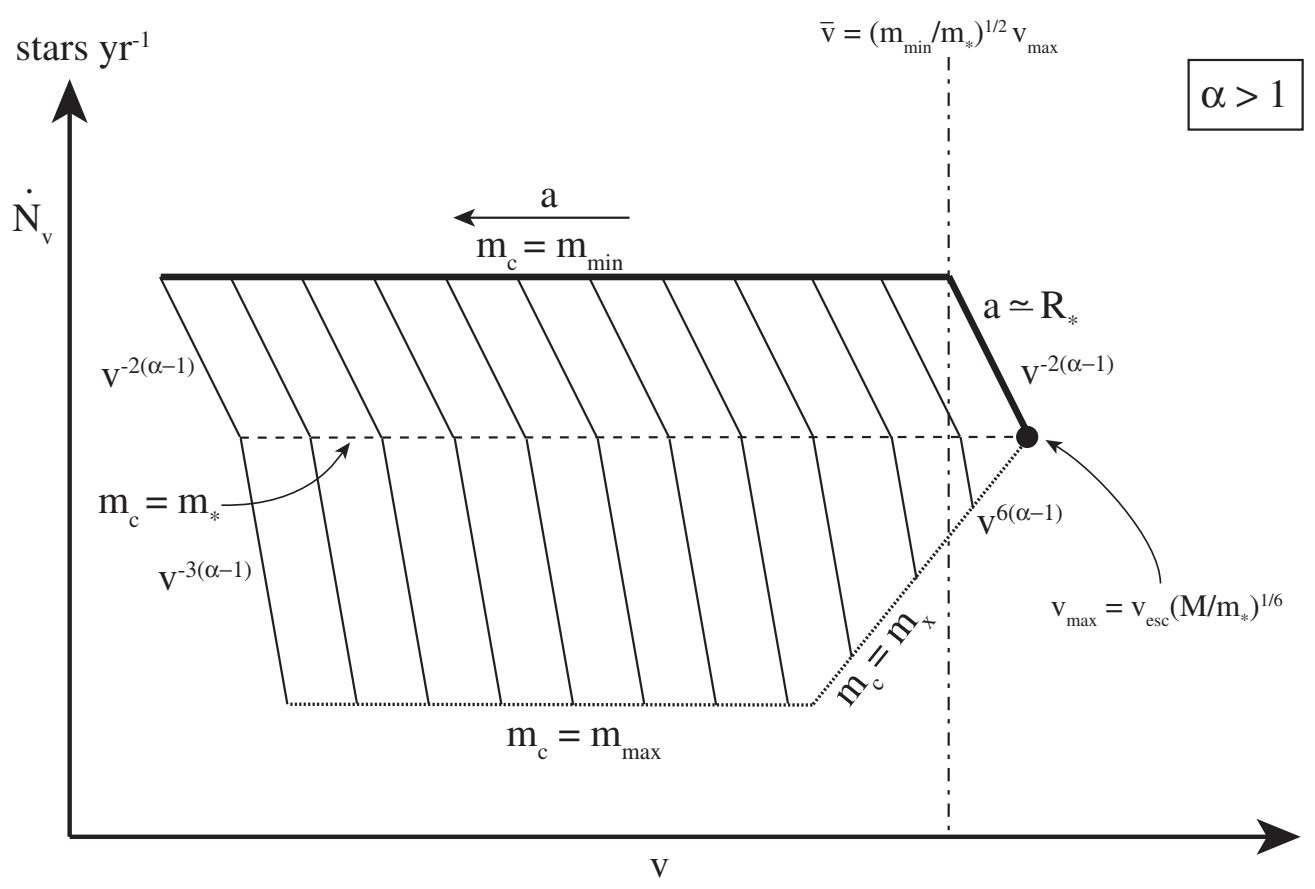

Figure 1. Ejection velocity distribution for a given HVS with mass $m_{*}$. Empty loss-cone regime. The plot is for $f_{\mathrm{m}} \propto m^{-\alpha}$, with $\alpha \geqslant 1$. The solid, thin, and dashed lines show $\dot{N}_{\mathrm{v}}$ for a fixed binary separation $a$ (Equation (6)). The rightmost one is for $a=a_{\min }$, and the binary separation $a$ increases leftward. These lines are equally logarithmically spaced since $f_{\mathrm{a}} \propto 1 / a$ (Equation (3)). The dashed line is $\dot{N}_{\mathrm{v}}$ for equal-mass binaries $m_{\mathrm{c}}=m_{*}$. The dotted lines mark $\dot{N}_{\mathrm{v}}$ for the most massive companion allowed for a given binary separation $a$ (Equations (9) and (10)). Finally, the thick black solid line is the total $\dot{N}_{\mathrm{v}}$ : the low-velocity branch is given by binaries with $m_{\mathrm{c}}=m_{\min }$, while the high-velocity branch corresponds to binaries with $a \approx R_{*}=a_{\min }\left(m_{\mathrm{c}} \ll m_{*}\right)$. The maximum velocity is obtained for a contact, roughly equal-mass binary (the black circle).

their Roche lobes and quickly merge (the exact consequence of mass transfer depends on the mass ratio, e.g., Vanbeveren et al. 1998). Considering that the less massive member has a much higher density, to avoid such Roche lobe overflow, the separation needs to be larger than $\sim R_{\mathrm{c}}+R_{\mathrm{c}}\left(m_{*} / m_{\mathrm{c}}\right)^{1 / 3}$ if $m_{\mathrm{c}}>m_{*}$ or $\sim R_{*}+R_{*}\left(m_{\mathrm{c}} / m_{*}\right)^{1 / 3}$ if $m_{\mathrm{c}}<m_{*}$. For binaries consisting of very different mass members (e.g., $m_{\mathrm{c}} \gg m_{*}$ ), the simple constraint on the separation quickly becomes $R_{\mathrm{c}}$ while in the realistic model it remains as $2 R_{\mathrm{c}}$. However, these estimates are still approximations since the stars change their shape, making it easier for mass transfer to occur (Eggleton 1983). We will impose $a>2.5 \max \left[R_{*}, R_{\mathrm{c}}\right]$ (e.g., Vanbeveren et al. 1998) when we numerically discuss velocity distributions in Section 6. Since $v \propto a^{-1 / 2}$, the highest possible velocity is expected to be reduced by a factor of $\sim 1.6$ or less. As we will see below, the highest velocity is achieved for $m_{*} \sim m_{\mathrm{c}}$, the correction factor for the underestimate of the minimum separation is even smaller $\sim \sqrt{2.5 / 2} \sim 1.1$.

Under the requirement $a>R_{\mathrm{c}}+R_{*}$, the maximum allowed mass for the companion, $m_{\mathrm{c}, \max }$, is the minimum between $m_{\max }$ and $m_{\mathrm{x}} \approx m_{*}\left(a / R_{*}\right)$. The transition between $m_{\mathrm{x}}$ and $m_{\max }$ occurs at $a \approx R_{\max }+R_{*}$. For $a<R_{\max }+R_{*}$, binaries with $m_{\mathrm{c}}=m_{\mathrm{x}}$ have their separation nearly equal to the sum of their radii, $a \approx R_{*}+R_{\mathrm{c}}$. We call them contact binaries: i.e., binaries that are on the verge of starting mass transfer. They produce the highest ejection velocities for a given separation,

$$
v\left(m_{\mathrm{x}}\right) \propto m_{\mathrm{x}}^{-1 / 6}, \text { for } m_{\mathrm{x}} \gg m_{*} .
$$

The maximum of all $v\left(m_{\mathrm{x}}\right), v_{\max }$, occurs for approximately equal-mass binaries: $m_{\mathrm{x}} \approx m_{*}$ and $a \approx R_{*}, v_{\max } \approx$ $v_{\text {esc }}\left(M / m_{*}\right)^{1 / 6}$, where $v_{\text {esc }} \approx \sqrt{G m_{*} / R_{*}}$ is the escape velocity from the surface of the HVS. The result that more massive companions $\left(m_{\mathrm{c}}>m_{*}\right)$ do not yield higher velocities is a direct consequence of the linear dependence assumed between the radius and the mass of a star. A somewhat shallower relation is obtained combining theory and observations: $R_{*} \propto m_{*}^{0.8}$ (Hansen et al. 2004). Even assuming this latter slope, the conclusion that the maximum velocity is for equal-mass binaries would remain unchanged, since it holds for any power-law index steeper than $2 / 3$.

The velocity probability distribution for contact binaries with massive companions, $m_{\mathrm{c}} \gg m_{*}$, can be derived considering the stars within an area of the parameter space $d m_{\mathrm{c}} \times d a$, $f_{\mathrm{v}} d v=f_{\mathrm{a}} f_{\mathrm{m}} d m_{\mathrm{c}} d a$, under the condition $a / R_{*} \simeq m_{\mathrm{c}} / m_{*}$,

$$
v \times\left. f_{\mathrm{v}}\right|_{m_{\mathrm{c}}=m_{\mathrm{x}}} \propto m_{\mathrm{x}}^{1-\alpha} \propto v^{6(\alpha-1)}, \quad m_{\mathrm{x}} \gg m_{*},
$$

where we used Equation (8), while for $m_{\mathrm{c}}=m_{\max }$, the relative number of stars is constant (Equation (7))

$$
v \times\left. f_{\mathrm{v}}\right|_{m_{\mathrm{c}}=m_{\max }} \propto v^{0}
$$

(see the dotted lines in Figure 1).

From Figure 1, it is evident that the total rate of stars $\dot{N}_{\mathrm{v}} \propto v \times f_{\mathrm{v}}$ ejected with a given velocity $v$ is dominated by binaries with the least massive companion possible that can give that velocity (thick solid lines),

$$
\dot{N}_{\mathrm{v}} \propto \begin{cases}v^{0}, & v \ll \tilde{v}, \\ v^{-2(\alpha-1)}, & v \gg \tilde{v},\end{cases}
$$

where $\tilde{v} \sim v_{\text {esc }}\left(m_{\min } / m_{*}\right)^{1 / 2}\left(M / m_{*}\right)^{1 / 6}=v_{\max }\left(m_{\min } / m_{*}\right)^{1 / 2}$. In (Equation (11)), the low-velocity tail is due to ejections from binaries with a low-mass companion $m_{\mathrm{c}}=m_{\min }$ on varying separations, while the high-velocity branch is given by dissolving contact binaries with $a \simeq R_{*}$ (i.e., $m_{\mathrm{c}} \ll m_{*}$ ). The 
maximum velocity $v_{\max }$ results from dissolving an equal-mass binary. Note that in Figure 1 there is a sharp cut-off at $v_{\max }$. This is a consequence of our approximate method: a full integration over all binaries that contribute to a given velocity would result in a more gradual decrease to zero of the velocity distribution.

\subsection{The Full Loss-cone Regime}

In the full loss-cone regime, the presence of the $\mathrm{BH}$ leaves the star distribution function almost unchanged and nearly isotropic at all $J$ (Lightman \& Shapiro 1977). Contrary to the empty losscone regime, this implies a disruption probability as a function of $a$ where looser binaries are disrupted more easily. More precisely, the cumulative probability to have $D \leqslant 1$ is

$$
P_{\mathrm{D} \leqslant 1} \propto r_{\mathrm{t}}
$$

The velocity probability density for a given binary semi-major axis is then given by $\left.f_{\mathrm{v}}(v)\right|_{a} d v=f_{\mathrm{m}}\left(m_{\mathrm{c}}\right) P_{\mathrm{D} \leqslant 1} d m_{\mathrm{c}}$, where we consider that only a fraction $P_{\mathrm{D} \leqslant 1}$ of binaries are disrupted. Therefore, $v \times\left. f_{\mathrm{v}}(v)\right|_{a} \propto m_{\mathrm{c}}^{-\alpha+1}\left(M / m_{\mathrm{t}}\right)^{1 / 3}$, and

$$
v \times\left. f_{\mathrm{v}}(v)\right|_{\mathrm{a}} \propto \begin{cases}v^{-2(\alpha-1)}, & m_{\mathrm{c}} \ll m_{*}, \\ v^{-3 \alpha+2}, & m_{\mathrm{c}} \gg m_{*},\end{cases}
$$

where we used Equation (5). As in the empty loss cone, the break occurs at the velocity for an equal-mass binary, $v\left(m_{\mathrm{c}}=m_{*}\right)$. The distribution is shown in Figure 2 as thin solid lines that correspond to different separations, which decrease rightward. For fixed masses, we instead have $\left.f_{\mathrm{v}}(v)\right|_{m_{\mathrm{c}}} d v=f_{\mathrm{a}} P_{\mathrm{D} \leqslant 1} d a$, and the rate at fix mass is

$$
v \times\left. f_{\mathrm{v}}(v)\right|_{m_{\mathrm{c}}} \propto a \propto v^{-2} .
$$

An example is shown by the dashed line in Figure 2 (upper panel) for $m_{\mathrm{c}}=m_{*}$. Finally, we derive the relative number of ejected stars associated with the maximum allowed mass, $m_{\mathrm{c}, \max }$ for a given separation. In the case of contact binaries, we follow the reasoning outlined in the previous section, taking into account the ejection probability. Since $f_{\mathrm{v}} d v=f_{\mathrm{a}} f_{\mathrm{m}} P_{\mathrm{D} \leqslant 1} d m_{\mathrm{c}} d a$, it follows

$$
v \times\left. f_{\mathrm{v}}\right|_{m_{\mathrm{c}}=m_{\mathrm{x}}} \propto m_{\mathrm{x}}^{5 / 3-\alpha} \propto v^{6 \alpha-10}, m_{\mathrm{x}} \gg m_{*},
$$

where we used Equation (8). If the binary is wide enough so that the more massive companion possible is $m_{\mathrm{c}}=m_{\max }$, then the velocity distribution is given by (Equation (14)),

$$
v \times\left. f_{\mathrm{v}}\right|_{m_{\mathrm{c}}=m_{\max }} \propto v^{-2} .
$$

Equations (15) and (16) are shown in Figure 2 as dotted lines.

Comparing the decay indexes of Equations (13) and (15) with -2 (Equation (14)), one realizes that there are three different regimes in which different types of binaries dominate the overall distribution.

For a typical stellar mass distribution with $\alpha>2$ (see Figure 2, upper panel), the main contribution to the total rate of ejected stars with a certain velocity $v, \dot{N}_{\mathrm{v}}$, comes from binaries whose companion has the least mass possible (i.e., the most abundant). This is analogous to the case $\alpha>1$, in the empty loss-cone regime, and

$$
\dot{N}_{\mathrm{v}} \propto \begin{cases}v^{-2}, & v<\tilde{v} \\ v^{-2(\alpha-1)}, & v>\tilde{v}\end{cases}
$$

where the low-velocity tail corresponds to binaries with companion mass $m_{\mathrm{c}}=m_{\min }$ and the high-velocity branch is due to contact binaries with $m_{\mathrm{c}} \ll m_{*}$ (thick solid lines in Figure 2, upper panel).

For a power-law index $4 / 3<\alpha \leqslant 2$ (see Figure 2, lower panel) the total rate (thick solid lines) is dominated at low velocities by wide binaries (with $a=a_{\max }$ and $m_{\mathrm{c}} \leqslant m_{*}$ ), while at high velocities is dominated by equal-mass binaries $\left(m_{\mathrm{c}}=m_{*}\right)$,

$$
\dot{N}_{\mathrm{v}} \propto \begin{cases}v^{-2(\alpha-1)}, & v<v\left(a_{\max }, m_{*}\right), \\ v^{-2}, & v>v\left(a_{\max }, m_{*}\right) .\end{cases}
$$

Since $a_{\max } \gg a_{\min }$, it is in fact likely that $v^{-2}$ is the only relevant slope for HVSs.

For a very shallow slope of index $\alpha<4 / 3$, most of the power at high velocities comes from contact binaries with $m_{\mathrm{c}} \gg m_{*}$ and the distribution follows $\dot{N}_{\mathrm{v}} \propto v^{6 \alpha-10}$.

The important result here is that the high-velocity slope is very steep, with a slope of -2 or steeper. Comparing our results with those of the previous section, we note that in both regimes $\dot{N}_{\mathrm{v}} \propto v^{-2.7}$ for a Salpeter mass function.

\subsection{Equal-mass Binaries}

In case binaries - especially massive ones - tend to be of equal mass, $\left(m_{\mathrm{c}}=m_{*}\right)$, the velocity distributions are given by the dashed lines in Figures 1 and 2. The relative number of stars in a velocity interval is constant in the empty loss cone (Equation (7)) and $\dot{N}_{\mathrm{v}} \propto v^{0}$. In the full loss-cone regime, instead, the relevant distribution is given by Equation (14), and the rate of ejected stars is $\dot{N}_{\mathrm{v}} \propto v^{-2}$. Interestingly, harder binaries have a smaller $J_{\mathrm{lc}}$ and could be in the full loss-cone regime, while wider binaries may not (Perets \& Gualandris 2010).

This would produce a broken power-law distribution from a flat slope to $v^{-2}$, where the break is at the separation $a$ where there is the transition between the two regimes.

\subsection{Summary of Our Analytical Results}

Above we derived the number of ejected stars produced by binaries of all separations and mass ratios. This allowed us to deduce the total velocity distribution, the one presented in Figures 1 and 2 by a solid thick line. Here we summarize its derivation.

First, we realize that the fastest possible ejection velocity is that obtained from a nearly equal mass, contact binary $\left(m_{*} \approx m_{\mathrm{c}}, a \approx 2.5 R_{*}\right)$,

$$
v_{\max } \approx 0.56 v_{\mathrm{esc}}\left(\frac{M}{m_{*}}\right)^{1 / 6} \approx 3500\left(\frac{m_{*}}{3 M_{\odot}}\right)^{-1 / 6} \mathrm{~km} \mathrm{~s}^{-1},
$$

where the numerical estimates are for $v_{\text {esc }}=\left(2 G m_{*} / R_{*}\right)^{1 / 2} \sim$ $600 \mathrm{~km} \mathrm{~s}^{-1}$ and $M=4 \times 10^{6} M_{\odot}$. If the binary separation distribution is truncated at some $a=k R_{*}$ (e.g., due to Roche lobe overflow), then $v_{\max }$ would be smaller by a factor of $(k / 2.5)^{1 / 2}$.

Velocities lower than $v_{\max }$, populating the high-velocity branch, can be obtained either by wider equal-mass binaries (e.g., Figure 2, lower panel), or by binaries with smaller companion masses but constant separation $a \approx 2.5 R_{*}$ (e.g., Figure 2, upper panel), whichever dominates. In this latter case, the relative frequency of ejected stars is simply given by the 

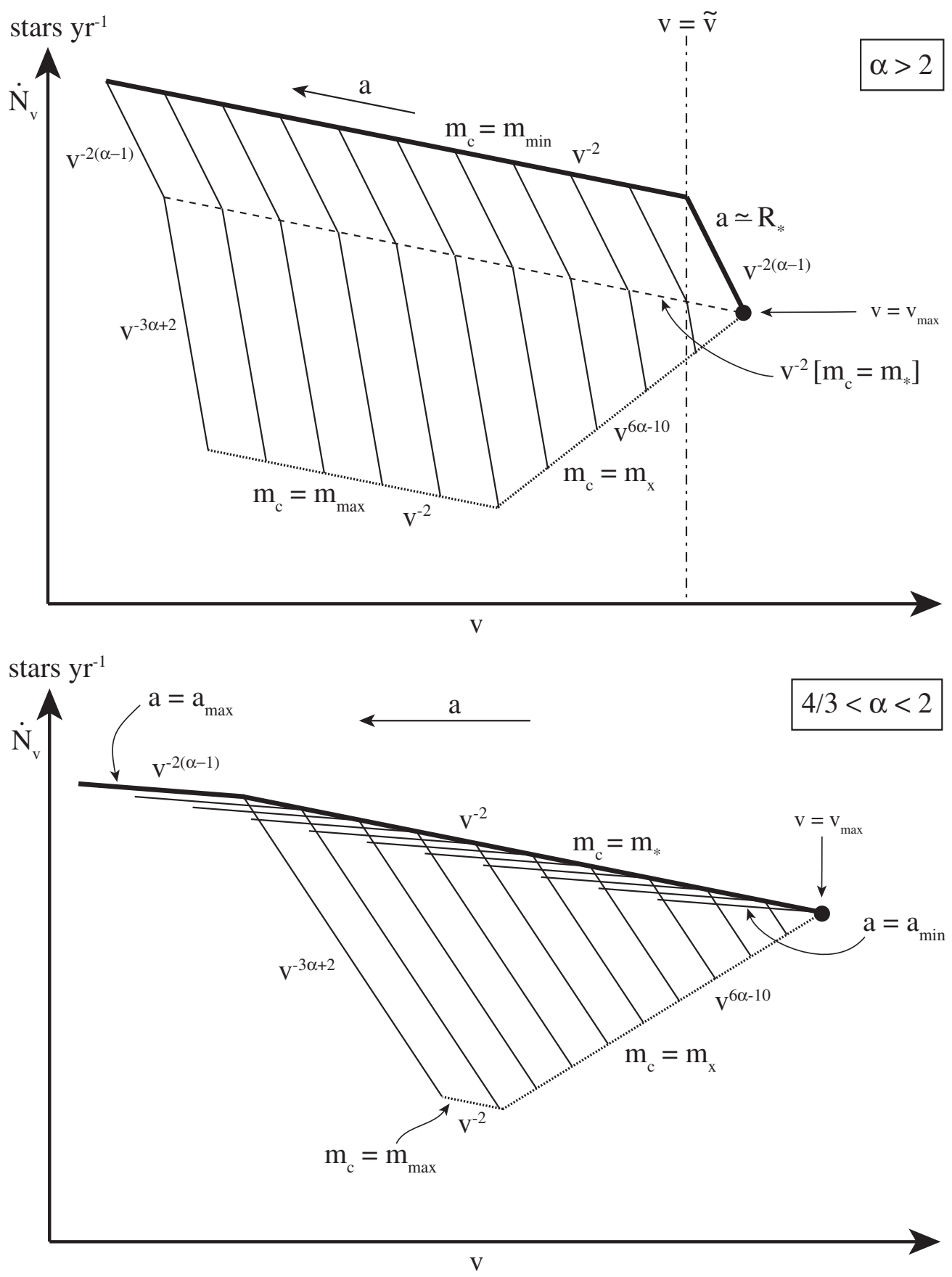

Figure 2. Same as Figure 1, but for the full loss-cone regime. The upper panel is for $\alpha \geqslant 2$. The high-velocity branch is for binaries with $a \simeq R_{*}$ and the slope is the same as for the empty loss-cone regime. The lower panel is for $4 / 3<\alpha<2$, and the high-velocity branch is populated by equal-mass binaries and the slope is shallower than in the upper panel case.

higher relative frequency of the lighter companions $\propto m_{\mathrm{c}}^{1-\alpha}$. Since the ejection velocity depends on lighter companions as $v \propto m_{\mathrm{c}}^{1 / 2}$, the velocity distribution has a slope of $v^{-2(\alpha-1)}$. On the other hand, the relative frequency of low-velocity HVSs that result from wider dissolved binaries depends on the circumstances. We have assumed $a \times f_{\mathrm{a}}=$ const., so wider binaries in general are just as common. In the empty loss-cone regime, where the rate of dissolving binaries is independent of the tidal radius, this leads to a flat distribution $v \times f_{\mathrm{v}}=$ const. However, in the full loss-cone regime, where the dissolving rate is proportional to the tidal radius, this leads to $v \times f_{\mathrm{v}} \propto r_{\mathrm{t}} \propto a \propto v^{-2}$.
Given a mass function where ${ }^{5} \alpha>2$, the lighter companion slope for a fixed separation $a$ is steeper than the slope for a fixed mass $m_{\mathrm{c}}$ (Figures 1 and 2, upper panel). The high-velocity distribution is therefore given by $v \times f_{\mathrm{v}} \propto v^{-2(\alpha-1)}$, down to the velocity produced by a binary with $a \approx 2.5 R_{*}$ and a minimal mass companion $m_{\min }$ :

$$
\tilde{v} \approx 0.63 v_{\mathrm{esc}}\left(\frac{M}{m_{\mathrm{t}}}\right)^{1 / 6}\left(\frac{m_{\min }}{m_{*}}\right)^{1 / 2} \approx 1580 \mathrm{~km} \mathrm{~s}^{-1} .
$$

\footnotetext{
5 In fact, already for $\alpha>1$ for the empty loss cone.
} 
The numerical evaluation is for the same values of Equation (19) and $m_{\min }=0.5 M_{\odot}$. Below this velocity, the distribution is either flat, $v \times f_{\mathrm{v}}=$ const. (empty loss cone) or $v \times f_{\mathrm{v}} \propto v^{-2}$ (full loss cone).

An implication of our results in both regimes is that, if $a_{\text {min }} \approx 2.5 R_{*}$, the high-velocity branch in case of $\alpha>2$ depends crucially on the existence and physical properties of contact binaries (i.e., binaries on the verge of starting mass transfer). Observationally, we lack firm constraints. It is not clear if contact binaries follow the same mass distribution as wider binaries, and if the minimal separation is indeed given by the mass transfer limit or is larger.

\section{VELOCITY DISTRIBUTION IN THE GALACTIC HALO}

HVSs are observed in the Galactic halo after they have traveled distances of several tens of kiloparsecs. The velocity distribution at such large distances from the $\mathrm{BH}$ can be derived from $\dot{N}_{\mathrm{v}}$, once we take into account the effects of the Galactic deceleration and of the finite lifetime of stars.

The former modifies the low tail velocities, below an effective escape velocity $v_{\mathrm{G}}$, where stars consume most of their initial energy to climb out of the potential well and reach the halo. Models for the Galactic potential predict that the escape velocity goes from $\sim 800 \mathrm{~km} \mathrm{~s}^{-1}$ at the radius of influence of the $\mathrm{BH}$ to $\sim 500-600 \mathrm{~km} \mathrm{~s}^{-1}$ in the solar neighborhood, ${ }^{6}$ and to $\sim 300-400 \mathrm{~km} \mathrm{~s}^{-1}$ in the halo at distances smaller than $80 \mathrm{kpc}$ (see model discussion in Kenyon et al. 2008 and Figure 3 in Brown et al. 2012b). In the following we will assume that stars are far out enough in the halo that most of their deceleration has already occurred. This is because most of the deceleration occurs just outside the sphere of influence of the BH (Kenyon et al. 2008), while most of the observed HVSs are at distances larger than $\sim 50 \mathrm{kpc}$. Since most of the deceleration occurs between a few parsecs and $\sim 100 \mathrm{pc}$, an effective Galactic escape velocity could be $v_{\mathrm{G}} \approx 800 \mathrm{~km} \mathrm{~s}^{-1}$.

We can thus relate the velocity at large distances $v_{\infty}$ to the velocity $v$, which takes only the $\mathrm{BH}$ potential into account, by $v_{\infty}=\sqrt{v^{2}-v_{\mathrm{G}}^{2}}$. It then follows that $v_{\infty} f_{\mathrm{v}_{\infty}}=v f_{\mathrm{v}}\left(v_{\infty} / v\right)^{2}$. Therefore, for any distribution $f_{\mathrm{v}}$, we obtain a strong lowvelocity cut-off $\propto v_{\infty}^{2}$ for $v_{\infty} \ll v_{\mathrm{G}}$. In particular, for power-law distributions $v f_{\mathrm{v}} \propto v^{-\beta}$, we obtain

$$
v_{\infty} f_{\mathrm{v}_{\infty}} \propto\left(v_{\infty}^{2}+v_{\mathrm{G}}^{2}\right)^{-(\beta+2) / 2} v_{\infty}^{2}
$$

which, for $v_{\infty} \ll v_{\mathrm{G}}$, recovers the $\propto v_{\infty}^{2}$ behavior, while for $v_{\infty} \gg v_{\mathrm{G}}$ has the original power-law slope, which is unaffected by the Galactic deceleration $\propto v_{\infty}^{\beta}$. We underline that this low-velocity break, including the shape around the break, is a robust prediction, since it does not depend on any of the binary distributions or on the injection mechanism (full versus empty loss cone). In the following, we will drop the subscript $\infty$ used for convenience to derive Equation (21), and $v$ should be understood as the velocity at infinity.

We are now in the position to compute the most relevant distribution to compare with observations: the number of stars with a given velocity $v$ within a distance $r$ from the $\mathrm{BH}$. At this aim, we need to account for the finite life of stars, $t_{*} \simeq 10^{10}\left(m_{*} / M_{\odot}\right)^{-2.5} \mathrm{yr}$ (Hansen \& Kawaler 1994), and the effect of propagation: $d N_{\mathrm{v}} / d r=\dot{N}_{\mathrm{v}} v^{-1}$, which tells us that slower stars are easier to observe at a given location. From

\footnotetext{
6 This is actually observed, e.g., Smith et al. (2007).
}

these considerations, the integrated quantity can be calculated as $N_{\mathrm{v}}(<r)=\dot{N}_{\mathrm{v}} \times \min \left[r / v, t_{*}\right]$. We conclude that the velocity distribution within $r$ has a break determined by both $r$ and the mass of the HVS,

$$
N_{\mathrm{v}}(<r) \propto \begin{cases}\dot{N}_{\mathrm{v}} & v<v_{\text {age }} \\ \dot{N}_{\mathrm{v}} v^{-1} & v>v_{\text {age }}\end{cases}
$$

HVSs, whose masses are $\sim 3-4 M_{\odot}$, are currently observed out to a distance of $\sim 100 \mathrm{kpc}$. More massive stars could in principle be observed even at larger radii. If we are considering distances within $r=100 \mathrm{kpc}$ and a massive star of $m_{*}=10 M_{\odot}$ $\left(m_{*}=3 M_{\odot}\right)$, the "age" break in the distribution occurs at $v_{\text {age }}=r / t_{*} \approx 3 \times 10^{3} \mathrm{~km} \mathrm{~s}^{-1}\left(v_{\text {age }}=r / t_{*} \approx 150 \mathrm{~km} \mathrm{~s}^{-1}\right)$. Note that we assume here a single age for all stars of a given mass. Brown et al. (2012a) estimate a $100 \mathrm{Myr}$ time lag between formation and ejections of stars in the observed sample. If taken into account, this would just cause a smoothing of the break over a very narrow range (a factor $\sim 1.18$ ) in velocities, and we ignore this correction. If the lag time is proportional to the star lifetime, then the correction can be equally ignored for any HVS mass.

When $v_{\text {age }} \ll v_{\mathrm{G}}$ (e.g., for $m_{*}=3 M_{\odot}$ ), the peak of the cumulative distribution $N_{\mathrm{v}}(<r)$ occurs at $v_{\text {peak }}=v_{\mathrm{G}} / \sqrt{1+\beta}$, while in the opposite case (e.g., for $\left.m_{*}=10 M_{\odot}\right)$, the peak is at $v_{\text {peak }}=v_{\mathrm{G}} / \sqrt{(\beta / 2)}$. For $m_{*}<10 M_{\odot}$ and equal-mass binaries, the value of the index $\beta$ depends on the loss-cone regime and on the binary separation distribution only. With our fiducial choice of $f_{\mathrm{a}}, \beta=0$ in the empty loss cone, and $\beta=2$ in the full loss cone. For more massive stars, $\beta=2(\alpha-1)$, with a dependence on the mass distribution only. An accurate description around the peak for $m_{*}=3 M_{\odot}$ is then given by

$N_{\mathrm{v}}(<r) \propto\left\{\begin{array}{lll}\frac{v}{v^{2}+v_{\mathrm{G}}^{2}} & v_{\text {peak }}=v_{\mathrm{G}} & \text { for empty loss cone }, \\ \frac{v}{\left(v^{2}+v_{\mathrm{G}}^{2}\right)^{2}} & v_{\text {peak }}=v_{\mathrm{G}} / \sqrt{3} & \text { for full loss cone. }\end{array}\right.$

For $m_{*}=10 M_{\odot}$ and a power-law distribution for the companion mass, irrespectively of the regime,

$$
N_{\mathrm{v}}(<r) \propto \frac{v^{2}}{\left(v^{2}+v_{\mathrm{G}}^{2}\right)^{\alpha}} \quad v_{\text {peak }}=v_{\mathrm{G}} / \sqrt{\alpha-1} .
$$

Finally, for $m_{*}=10 M_{\odot}$ and equal-mass binaries, we have:

$$
N_{\mathrm{v}}(<r) \propto\left\{\begin{array}{lll}
\frac{v^{2}}{v^{2}+v_{\mathrm{G}}^{2}} & \text { no clear break } & \text { for empty loss cone }, \\
\frac{v^{2}}{\left(v^{2}+v_{\mathrm{G}}^{2}\right)^{2}} & v_{\text {peak }}=v_{\mathrm{G}} & \text { for full loss cone. }
\end{array}\right.
$$

Besides the peak velocity, Equation (22) implies two other breaks for unequal-mass binaries, when $m_{*}<10 M_{\odot}$ : at $v_{\text {age }}$ and $\tilde{v}$ (ejection velocity imparted when a contact binary contains the lightest companion in the distribution; Equation (20)). For larger HVS mass and for equal-mass binaries, instead, there is no break at $\tilde{v}$ and there are only three power-law branches instead of four. The relative order of these characteristic velocities at a given distance " $r$ " depends on the HVS mass. For a $m_{*}=3 M_{\odot}$, $v_{\text {age }} \approx 150 \mathrm{~km} \mathrm{~s}^{-1}<v_{\text {peak }}<\tilde{v} \approx 1580 \mathrm{~km} \mathrm{~s}^{-1}<v_{\max } \approx$ $3500 \mathrm{~km} \mathrm{~s}^{-1}$. For $m_{*}=10 \mathrm{M}_{\odot}$, the break ordering is different, $v_{\text {peak }} \approx 690 \lesssim \tilde{v} \approx 720 \mathrm{~km} \mathrm{~s}^{-1}<v_{\text {age }} \approx 3000 \mathrm{~km} \mathrm{~s}^{-1}$, and a smaller maximum velocity is attained $v_{\max } \approx 2900 \mathrm{~km} \mathrm{~s}^{-1}$.

In the following, we give examples of distributions for $m_{*}=3 M_{\odot}$ and $m_{*}=10 M_{\odot}$, assuming a Salpeter mass 
function for the companion $m_{\mathrm{c}}$. For $m_{*}=3 M_{\odot}$, in the empty loss-cone regime, the distribution is

$$
N_{\mathrm{v}}(<r) \propto \begin{cases}v^{2} & v<v_{\text {age }}, \\ v & v_{\text {age }}<v<v_{\text {peak }}, \\ v^{-1} & v_{\text {peak }}<v<\tilde{v} \\ v^{-3.7} & \tilde{v}<v<v_{\max },\end{cases}
$$

where $v_{\text {peak }}=v_{\mathrm{G}} \sim 800 \mathrm{~km} \mathrm{~s}^{-1}$. We note that there is a remarkable steepening after $\tilde{v}$, which makes observations of HVSs with extreme velocities quite unlikely. For a full loss cone, the slope is instead quite steep all the way from the peak, inhibiting detections of HVSs with velocity above a few times the peak velocity $\left(\gtrsim 10^{3}\right) \mathrm{km} \mathrm{s}^{-1}$,

$$
N_{\mathrm{v}}(<r) \propto \begin{cases}v^{2} & v<v_{\text {age }}, \\ v & v_{\text {age }}<v<v_{\text {peak }}, \\ v^{-3} & v_{\text {peak }}<v<\tilde{v}, \\ v^{-3.7} & \tilde{v}<v<v_{\max },\end{cases}
$$

where $v_{\text {peak }}=v_{\mathrm{G}} / \sqrt{3} \sim 460 \mathrm{~km} \mathrm{~s}^{-1}$. For $m_{*}=10 M_{\odot}$, the velocity distribution is

$$
N_{\mathrm{v}}(<r) \propto \begin{cases}v^{2} & v<v_{\text {peak }}, \\ v^{-2.7} & v_{\text {peak }}<v<v_{\text {age }}, \\ v^{-3.7} & v_{\text {age }}<v<v_{\text {max }} .\end{cases}
$$

in both loss-cone regimes. Here, a sharp steepening occurs right after the peak $v_{\text {peak }}=v_{\mathrm{G}} / \sqrt{1.35} \sim 700 \mathrm{~km} \mathrm{~s}^{-1}$.

\section{MONTE CARLO CALCULATIONS}

We perform Monte Carlo simulations to check whether our analytic results can indeed capture the main features of the distributions. In particular, we investigate the effect of starstar collisions, and how our assumptions on the energy and probability distribution affect our results at the high-velocity end. The large mass ratio $M / m_{\mathrm{t}} \gg 1$ allows us to formulate the problem of a binary-supermassive $\mathrm{BH}$ encounter in a restricted three-body approximation, and the ejection velocity is given by

$$
v=\sqrt{\frac{2 G m_{\mathrm{c}}}{a}}\left(\frac{M}{m_{\mathrm{t}}}\right)^{1 / 6} \bar{E}^{1 / 2},
$$

where the (dimensionless) energy gain $\bar{E}$ at the disruption depends on the geometry of the encounter and should be computed numerically (see SKR). In our analytical calculations, we have assumed a simple model where only and all binaries approaching the $\mathrm{BH}$ with $D \leqslant 1$ are disrupted and $\bar{E}=1$. In our Monte Carlo calculations, we instead numerically integrate the orbit of each realization to determine whether the binary survives the encounter and, in case it is disrupted, we calculate the actual energy gain $\bar{E}$. Finally-unlike in the analytic treatment-we can take into account the finite size of stars and determine whether binary members collide under the tidal force, instead of being torn apart.

\subsection{Procedure}

The initial position and velocity of the binary center of mass are chosen so that the binary approaches a massive $\mathrm{BH}$ in a parabolic orbit. The encounter of a circular binary with the $\mathrm{BH}$ is characterized by nine parameters: masses of the $\mathrm{BH}$ and binary members: $M, m_{*}$, and $m_{\mathrm{c}}$, binary separation $a$, penetration factor $D$, binary plane orientation $(\theta, \varphi)$, and binary phase $\phi$ at the initial distance $r_{0}$. As long as a simulation starts at a large enough radius $r_{0} \gg r_{\mathrm{t}}$, the results are largely independent of it. Since we consider HVSs with $m_{*}=3 M_{\odot}$ or $10 M_{\odot}$ ejected from a massive $\mathrm{BH}$ with $M=4 \times 10^{6} M_{\odot}$, we have at most six random variables in our Monte Carlo simulations: $m_{\mathrm{c}}, D, \theta, \varphi$, $\phi$, and $a$.

For each realization, we numerically integrate the evolution of the binary based on the restricted three-body approximation. More precisely, we use Equations (8)- (10) and (12) in SKR. The numerical code is provided with a fourth-order Runge-Kutta integration scheme. We set our initial conditions at $r_{0}=10 r_{\mathrm{t}}$. There, we assign a penetration factor $D$, an initial binary phase $\phi$, and the binary plane direction, defined by the two angles $\theta$ and $\varphi$. There are two special inclinations: prograde binaries, whose spins are aligned with the orbital angular momentum of the center of mass, and retrograde binaries, whose spins are instead counter-aligned.

We assign $D$ and the various angles as follows. In the empty loss-cone regime, we assume that $D$ is uniformly distributed between zero and $D_{\max }=2.1$, where $D_{\max }$ is the largest value for which disruption can occur for circular binaries with any binary plane inclination. ${ }^{7}$ In fact, for each binary system, there is a different minimum $D$ that a binary can reach without one of the stars being tidally disrupted, $D \sim R / a$, where $R$ is the stellar radius of the more massive star. This is generally $\ll 1$; however, it can become of the order of $\sim 1$ for contact binaries. Modifications of the high-speed branch due to stellar disruptions will be important if/when speeds in excess of $1000 \mathrm{~km} \mathrm{~s}^{-1}$ will be measured. Lacking observational motivation, we will omit this effect in this paper. In the full loss-cone regime, we randomly choose the pericenter distance $r_{\mathrm{p}}$, instead of $D$, between 0 and $D_{\max } r_{\mathrm{t}, \max }$, where the maximum tidal radius is $r_{\mathrm{t}, \max }=a_{\max }\left[M /\left(m_{*}+m_{\min }\right)\right]^{1 / 3}$. This can be translated into the dimensionless distance $D$ once we assign a mass for the companion and a binary separation (see below). We also randomly choose the binary plane orientation $(\theta, \varphi)$ and the binary phase $\phi$ between 0 and $\pi$. Since a binary starting with a phase difference $\pi$ has the same post-encounter energy in absolute value but opposite in sign (SKR), the absolute values of the energies provide the ejection velocities for the whole range $0<\phi<2 \pi$.

If the numerical result indicates that the binary survives without disruption or that the minimal star separation during the evolution is smaller than the sum of the star radii (i.e., collision), the realization would be disregarded and another one produced until we have an ejection. The removal of colliding binaries prevents us from including spurious, very high-velocity events in the velocity distribution.

The analytical part of the ejection velocity depends solely on the companion mass and on the binary separation. We consider two mass distributions for $m_{\mathrm{c}}$ : the Salpeter mass function, $f_{\mathrm{m}} d m_{\mathrm{c}} \propto m_{\mathrm{c}}^{-2.35} d m_{\mathrm{c}}\left(0.5 M_{\odot} \leqslant m_{\mathrm{c}} \leqslant 100 M_{\odot}\right)$, and an equal-mass distribution: $m_{\mathrm{c}}=m_{*}$. The binary separation is assumed to be distributed uniformly in the logarithmic space: $f_{\mathrm{a}} d a \propto d a / a$ with a maximum separation given by the mean distance of stars in the sphere of influence of the $\mathrm{BH}$ : $a_{\max } \sim n_{*}^{-1 / 3} \sim 10^{6} R_{\odot} \sim 2 \times 10^{-2}$ pc where $n_{*} \approx 10^{5} \mathrm{pc}^{-3}$ is the mean stellar number density. We impose a minimum separation roughly equal to the distance at which the Roche

\footnotetext{
$7 \quad D_{\max }$ occurs for planar prograde orbits (SKR), which are the easiest configurations to dissolve.
} 


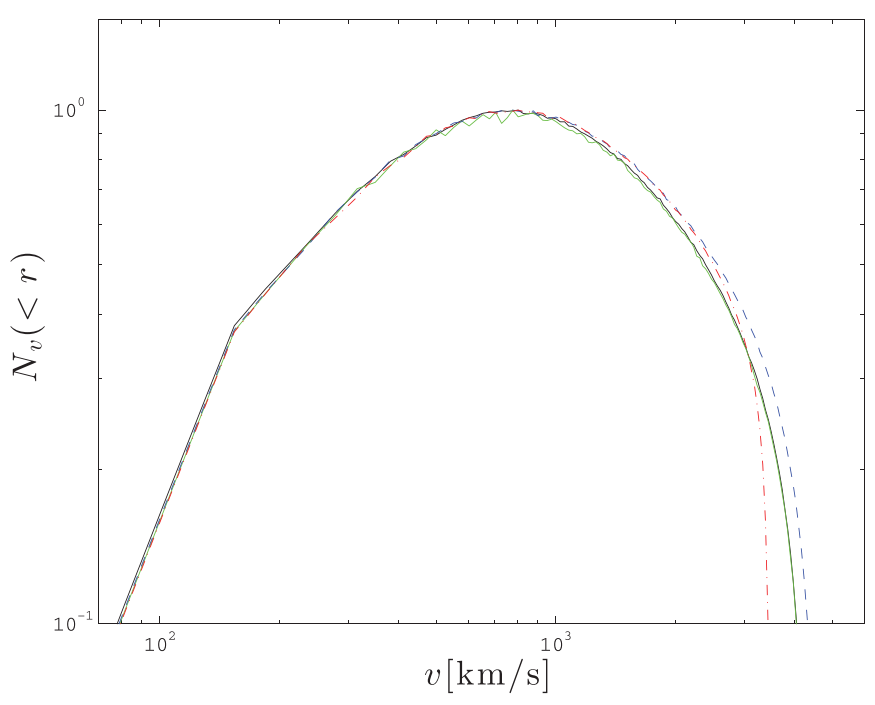

Figure 3. Study of our numerical results. We show the case of equal-mass binaries with $m_{*}=3 M_{\odot}$. The solid black and green lines are for the $10^{7}$ and $3 \times 10^{6}$ realizations, respectively. The blue dashed line is the velocity distribution if star-star collisions are not taken into account. Finally, the red dashed line shows the effect of assuming $\bar{E}=1$.

(A color version of this figure is available in the online journal.)

overflow will start $a_{\min }=2.5 \max \left[R_{*}, R_{\mathrm{c}}\right]$. However, we do not directly use this distribution. To more efficiently evaluate the high-velocity tail, we first adopt a steeper distribution, $f_{\mathrm{a}} d a \propto a^{-2} d a\left(R_{*}<a<10^{6} R_{\odot}\right)$. This function produces high-velocity events with higher frequency, since $v \propto a^{-1 / 2}$. To reconstruct a velocity distribution that reflects an $f_{\mathrm{a}} \propto 1 / a$ function, we then correct our histogram by counting " $a$ " times each realization in a velocity bin, produced by a certain " $a$."

The results in this paper are produced with $10^{7}$ realizations. A resolution study is shown in Figure 3, where the two velocity distributions computed with $10^{7}$ (black solid line) and $3 \times 10^{6}$ (green solid line) are indistinguishable, especially at high velocities.

\subsection{Results: Velocity Distributions}

Our results are shown in Figures 4 and 5 for the empty and full loss-cone regimes, respectively. In these figures, we plot the expected velocity distribution within $100 \mathrm{kpc}^{8}$ from the Galactic center for HVSs with $m_{\star}=3 M_{\odot}$ (upper panels) and $m_{\star}=10 M_{\odot}$ (lower panels). The distributions with a Salpeter mass function for the companion star are shown with red lines, while blue lines are for the equal-mass binaries. Our computational method enhances the statistics of the highvelocity branches (see Section 6.1) and comparatively gives larger statistical errors at low velocities. Furthermore, the Monte Carlo calculations first provide the velocity distribution in terms of the ejection velocity. When this is converted to the velocity at infinity, a narrow velocity region just above the escape velocity spans a wide region in the logarithmic space. To resolve the lowvelocity tail $\left(0<v<v_{\mathrm{G}}\right)$, we would need many velocity bins in the numerical calculations and consequently a much larger number of random realizations. However, since the velocity distribution is expected to be smooth in the narrow velocity region around $v_{\mathrm{G}}$, we have used a linear fit to the numerical data

\footnotetext{
8 Distance choices affect only the estimate of the age break $v_{\text {age }}$ in the figures. For a different distance, the break is shifted according to $v_{\text {age }} \sim 150(r / 100 \mathrm{kpc})\left(m_{*} / 3 M_{\odot}\right)^{2.5} \mathrm{~km} \mathrm{~s}^{-1}$.
}
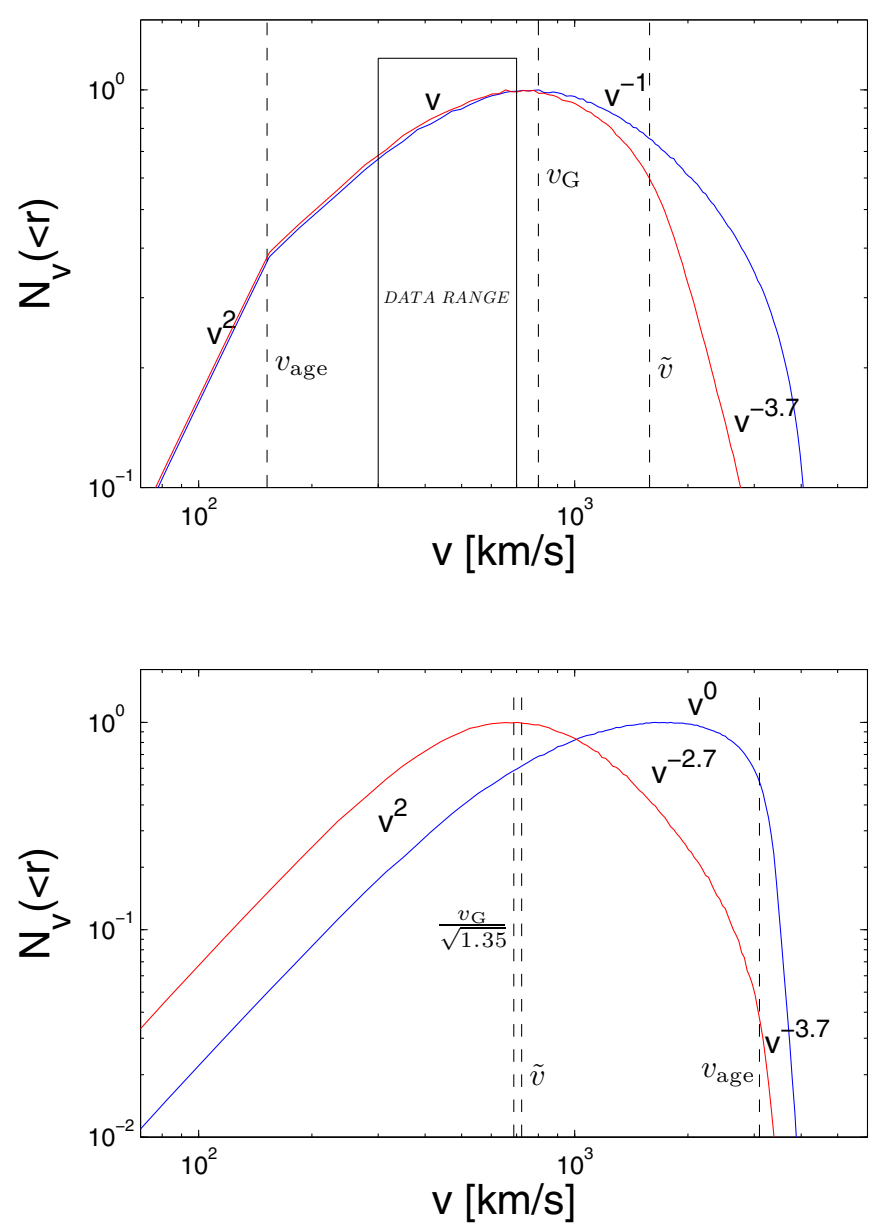

Figure 4. Relative number of stars with a given velocity, observed within a radius of $100 \mathrm{kpc}$ : empty loss-cone regime. In the upper panel $m_{*}=3 M_{\odot}$, while in the lower panel $m_{*}=10 M_{\odot}$. In each panel, the red line is for a Salpeter mass distribution for the companion star and the blue line is for equal-mass binaries. The distributions are all normalized at their peak. Vertical dashed lines mark the characteristic break velocities. The marked slopes are the analytical expectations (Section 5), which well describe our results (see the text for discussion). Toward $v_{\max }\left(\gtrsim 3000 \mathrm{~km} \mathrm{~s}^{-1}\right.$ in the plot), the slopes get much steeper than expected as the distribution will eventually become zero.

(A color version of this figure is available in the online journal.)

to construct the low-velocity tail. In contrast, the distribution for velocities $v>185 \mathrm{~km} \mathrm{~s}^{-1}$ comes directly from our numerical results.

Each part of the distribution encodes different information. The mass distribution function affects just the highest velocity branch, beyond $\tilde{v}$. This is because the main contribution to these velocities comes from binaries with same separation $\left(a_{\min }\right)$ but different companion masses. In our examples, a Salpeter mass function (red lines) results in a significantly steeper slope than for equal-mass binaries (blue lines). Shallower power-law slopes than the Salpeter one (but greater than 1) would result in intermediate velocity curves, between the blue and the red lines. The slope of the distribution at the right of the peak (between $\sim v_{\mathrm{G}}$ and $\tilde{v}$ ) is determined by the binary separation distribution, since the main contribution to this branch comes from binaries with the same star masses but different separations. As an example, a flatter distribution (e.g., $f_{\mathrm{a}}=$ const.) than a $f_{\mathrm{a}} \propto 1 / \mathrm{a}$ would have more power at larger separation and therefore would comparatively enhances lower velocities $\left(v \propto a^{1 / 2}\right)$, producing a steeper slope in the velocity distribution (we will use this feature when comparing our model with data in the next section). 

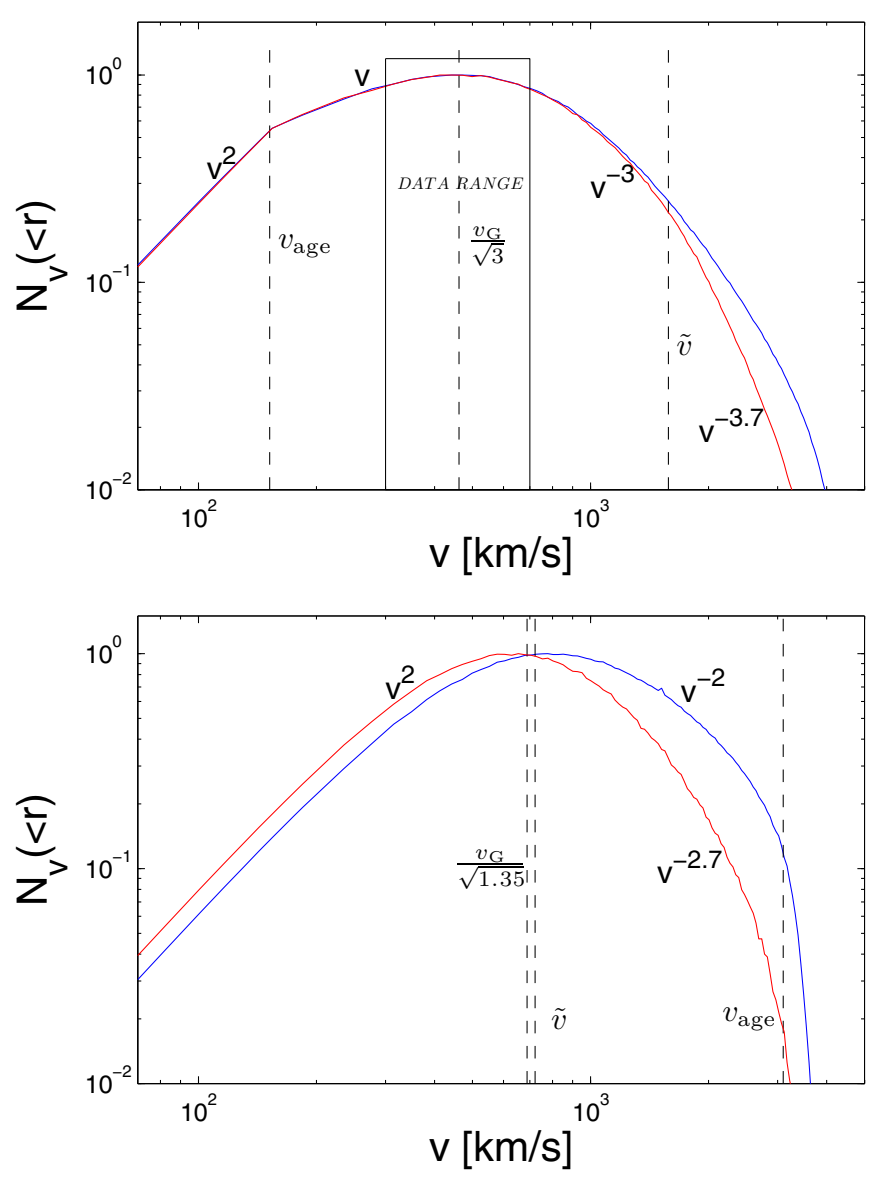

Figure 5. Same as Figure 4, but for a full loss-cone regime. (A color version of this figure is available in the online journal.)

Finally, before the peak, the distribution is determined by the Galactic deceleration.

The positions of the breaks bear important information as well. The peak velocity is a measure of the Galactic escape velocity, and value of $\tilde{v}$ is related to the mass of the lighter possible companion and $v_{\text {age }}$ is due to the HVS lifetime. However, while the peak velocity may be easy to determine, since we necessarily expect more events there, $\tilde{v}$ and $v_{\text {age }}$ may not. For example, in our Figures 4 and 5, the break related to $v_{\text {age }}$ is below $200 \mathrm{~km} \mathrm{~s}^{-1}$ for a $m_{\star}=3 M_{\odot}$. At these velocities, the number HVSs constitute only a tiny fraction of the "background" halo stars, whose Gaussian velocity distribution dominates for $|v|<300 \mathrm{~km} \mathrm{~s}^{-1}$. For a $m_{\star}=10 M_{\odot} \mathrm{HVS}$, the age break occurs at very high velocities $\left(\sim 3000 \mathrm{~km} \mathrm{~s}^{-1}\right)$, where, in all cases but one, the slopes are very steep, inhibiting detections. The break associated with $\tilde{v}$ is again beyond the velocity peak for $m_{*} \gtrsim 10 M_{\odot}$ and it is not present at all beyond this mass.

The above features were already understood from our analytical calculations. These give a good qualitative description of the velocity distribution (see Section 5) but our numerical results show differences, especially for the high-velocity branches beyond the peak. In our analytical treatment we assumed $\bar{E}=1$ and constant disruption probability for $D<1$. The mean dimensionless energy distribution shows that mean energies (averaged over all angles) $\langle\bar{E}\rangle$ can instead be larger than unity for milder penetrations $0.1<D<1$. The maximum is $\langle\bar{E}\rangle \approx 1.5$ at $D \approx 0.4$. In this range of $D$, the energy dispersion can also be substantial, becoming of the order of unity at $D \approx 0.1$. The consequence is that larger ejection velocities can be obtained and the maximum velocity is larger by a factor of $\sim \sqrt{1.5}$ with respect to our analytical expectations (Equation (19)). For instance, for $m_{*}=3\left(m_{*}=10\right)$, this implies $v_{\max } \approx 4300 \mathrm{~km} \mathrm{~s}^{-1}$ $\left(v_{\max } \approx 3500 \mathrm{~km} \mathrm{~s}^{-1}\right)$. On the other hand, the presence of a non-negligible dispersion in dimensionless energy causes the slopes to be a bit shallower for the highest velocities, and any break beyond $\tilde{v}$ to be smoother than predicted analytically. This is shown, for example, in Figure 3 with a comparison between the solid black (full calculation) and red dashed $(\bar{E}=1)$ lines. Our numerical calculations also show that the average disruption probability (averaged over all inclinations and phases) is quite constant for $D<0.1$, after which it decreases. It is $\approx 0.5$ at $D=1$. This feature makes ejections with $\bar{E}>1$ less frequent, mitigating the effect described above. In other words, if a constant disruption probability was imposed for all $D$ s, there would be an enhancement of high velocities and the resulting velocity distribution would, in fact, be more different from the analytical expectations at the high-velocity end. Finally, we numerically took into account collisions when the distance between stars becomes smaller than the sum of the two radii. In Figure 3, we show that if not excluded, those realizations yield artificially high ejection velocities (blue dashed line).

\subsection{Comparison with Current Data}

Current HVS surveys select HVSs with masses of 3-4 $M_{\odot}$. Up to now, $\sim 18$ HVSs have been observed within $\sim 100 \mathrm{kpc}$, with radial velocities ranging between 300 and $700 \mathrm{~km} \mathrm{~s}^{-1}$ (e.g., Brown et al. 2012b). Around $300 \mathrm{~km} \mathrm{~s}^{-1}$, there is an equal number of detected stars that have been classified as bound HVSs: they are thought to share the same physical origin as HVSs, but their velocity does not exceed the local Galactic escape speed. Below $300 \mathrm{~km} \mathrm{~s}^{-1}$, the population of stars bound to the halo dominates the velocity distribution and discovery of HVSs with only radial velocity measurements may become quite challenging. Beyond $720 \mathrm{~km} \mathrm{~s}^{-1}$, instead, there are no detections at all, and there is no obvious observational bias that explains it. For our purposes, we will consider only the unbound sample of $\sim 18$ stars, which has a narrow range of observed velocities $\left(\sim 380\right.$ to $\left.\sim 720 \mathrm{~km} \mathrm{~s}^{-1}\right)$ in which the lower end tends to be more populated than the upper end (see the histogram in Figure 6).

We should note that current observations measure only the radial component of the star velocity; thus, the true threedimensional velocity can be larger. However, if the observed stars are actually produced in the Galactic center, their trajectories at such large distances-much larger than the Sun distance from the Galactic center - should be almost radial as seen from the Sun. Therefore, we do not expect that a large correction should be applied to our predicted velocities, in order to compare our distributions with observations.

Theoretical arguments suggest that our Galactic center favors a situation in which the loss cone is empty. Massive binaries are observed to have periods that are flat in logarithmic scale (e.g., Kiminki \& Kobulnicky 2012). In these conditions, the predicted velocity distribution to compare with data is shown in Figures 6 and 7 as a red solid line (see also Figure 4, upper panel). Encouragingly, the range of observed velocities lies close to our peak velocity $\left(v_{\mathrm{G}}=800 \mathrm{~km} \mathrm{~s}^{-1}\right)$, in correspondence with the wide peak of the distribution. However, the data are not including the peak velocity (Figure 6), while the model would predict that velocities beyond the peak should be as common as those below it. In Figures 6 and 7, we also plot our fiducial 


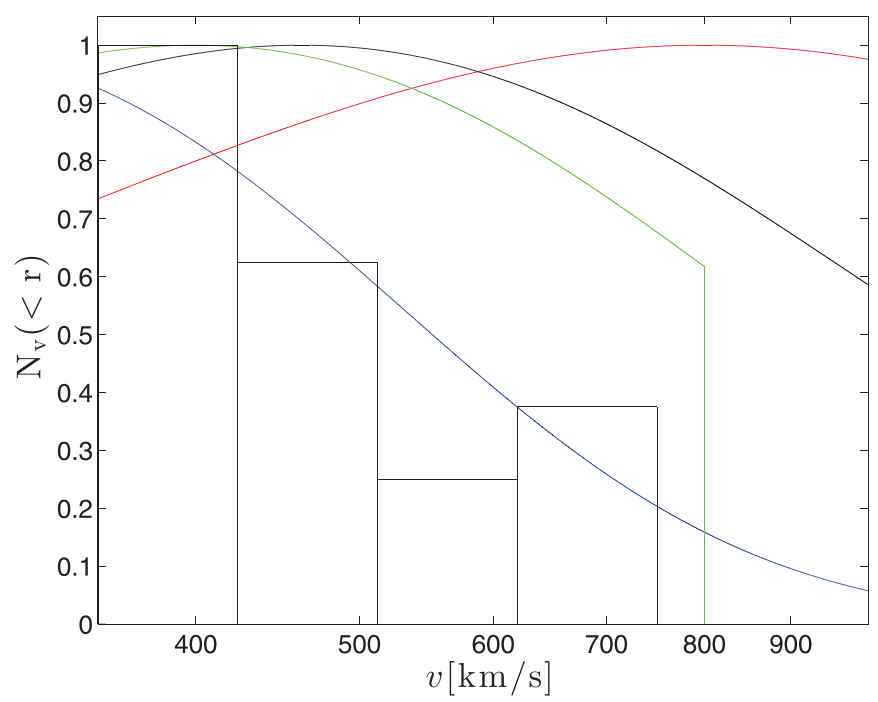

Figure 6. Relative number of stars with a given velocity, observed within a radius of $100 \mathrm{kpc}$ for a $3 M_{\odot}$ star. Distributions $N_{\mathrm{v}}(r<) \propto v f_{\mathrm{v}} \propto v /\left(v^{2}+v_{\mathrm{G}}^{2}\right)^{-(\beta+2) / 2}$ are normalized at the peak $\left(=v_{\mathrm{G}} / \sqrt{1+\beta}\right)$. The red and black line are for our fiducial model for an empty $(\beta=0)$ and full $(\beta=2)$ loss cone, respectively. The green line assume $\beta=3$ and $a_{\min }=10 R_{*}\left(\right.$ instead of $\left.a_{\min }=R_{*}\right)$, while the blue line is for $\beta=8$. Data in the histogram are taken from Table 1 in Brown et al. (2012b). The height of each bin indicates the fraction of HVSs in that bin relative to the peak. The bins have the same width in the log space.

(A color version of this figure is available in the online journal.)

model for a $3 M_{\odot}$ HVS, in the full loss-cone regime of star replenishment (black lines, see also Figure 5, upper panel). Although in this case when the peak $\approx 460 \mathrm{~km} \mathrm{~s}^{-1}$ is in the middle of the observed range, the model suffers from the same deficiencies, predicting $40 \%$ of HVSs with velocities higher than $>750 \mathrm{~km} \mathrm{~s}^{-1}$.

We must add here that observed HVSs may still be decelerating in the Galactic halo at the observed location, while our model calculates the final coasting velocities. Using various Galactic potential models from Kenyon et al. (2008), we found that a few (up to half) of the HVSs can indeed have lower final velocities, but that they remain distributed in the same range $300-700 \mathrm{~km} \mathrm{~s}^{-1}$. Lower velocities, however, can only strengthen our conclusion that both our fiducial models fail to account for current observations.

Analytically, the shape around the peak can be reproduced by the function $\propto v /\left(v^{2}+v_{\mathrm{G}}^{2}\right)^{-(\beta+2) / 2}$, which has a peak at $v_{\mathrm{G}} /(\sqrt{\beta+1})$. In our fiducial models, which overpredict high velocities, $\beta=0$ or $\beta=2$. We therefore need steeper velocity distributions in the observed range: i.e., larger $\beta$ values. We obtain possible $\beta$ values by comparing our model with the observed cumulative distribution (Figure 7). The blue line is for $\beta=8$ and the green line is for $\beta=3$ plus a sharp cutoff at $v \approx 800 \mathrm{~km} \mathrm{~s}^{-1}$. They both have a probability $\leqslant 10 \%$ for velocities higher than $>750 \mathrm{~km} \mathrm{~s}^{-1}$. The corresponding differential distributions are shown in Figure 6, with the same color scheme. The $\beta=8$ curve has a peak around $270 \mathrm{~km} \mathrm{~s}^{-1}$, fully embedded-and therefore hidden-in the halo stellar distribution.

What might these distributions mean for our binary/Galaxy physical parameter? As discussed in Section 5, the shape around the peak of the distribution for $m_{*}=3 m_{\odot}$ depends on three physical ingredients. (1) The value of the escape velocity, (2) the binary separation distribution, and (3) the injection mode of binaries into the tidal sphere of influence. Since the escape

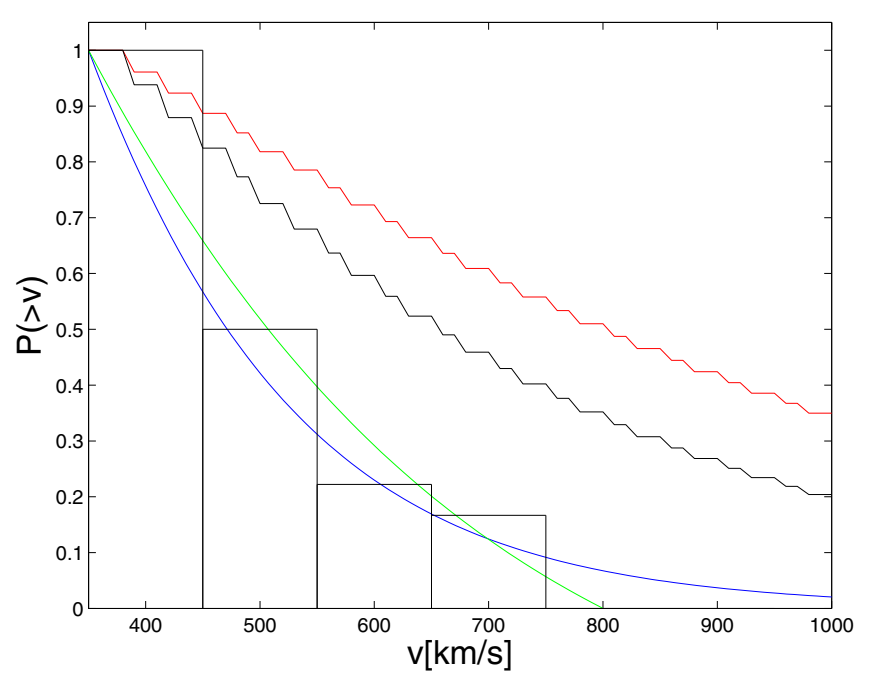

Figure 7. Same as Figure 6, but for the cumulative distributions. This time the fiducial models are plotted using the Monte Carlo data, and not the analytical approximation as in Figure 6: they are clearly not consistent with the cumulative distribution of the current sample. The cumulative histogram has the same bin width in the liner space.

(A color version of this figure is available in the online journal.)

velocity does not influence the value of $\beta$, we may assume that a steeper slope may be given by a combination of the last two points. Both an empty and full loss cone require a binary separation distribution that rises toward larger separations or at most decreases slowly to give more weight to lower velocities than to higher ones. In particular, an empty loss cone requires a binary separation distribution that rises as $f_{\mathrm{a}} \propto a^{3}$ (for $\beta=8$ ), while the green line in Figure 6 can be reproduced with $f_{\mathrm{a}} \propto a^{1 / 2}$ and a minimum separation for the binaries of $a_{\min } \approx 10 R_{*}$ (equal to $30 R_{\odot}$ in this case). A full loss cone already favors wide binaries over tight ones, and therefore requires shallower separation distributions: $f_{\mathrm{a}} \propto a^{2}$ and $f_{\mathrm{a}} \propto a^{-1 / 2}$. We may speculate on the origin of these distributions. If in the Galactic central bulge binaries had a separation distribution that peaks $\gg 1 \mathrm{AU}$ (or, equivalently, a period $\gg 30$ days), then the shorter period binaries may indeed be described by a rising powerlaw distribution in separations. To cite a well-known example, binaries in the solar neighborhood have a log-normal distribution in periods that peak at $10^{5}$ day (Duquennoy \& Mayor 1991). A possible cut-off at $\sim 10 R_{*}$ may instead be related to the binary formation/evolution process or to the injection mechanism which somehow filters out tight binaries.

The velocity distributions of HVSs in the Galactic halo have been investigated by a few groups through Monte Carlo simulations based on full three-body calculations (e.g., Bromley et al. 2006; Kenyon et al. 2008; Zhang et al. 2010, 2013). Zhang et al. (2010) is especially relevant to our study, and they have shown that the velocity distribution is sensitive to how binaries move into the $\mathrm{BH}$ loss cone, and that if binaries approach the $\mathrm{BH}$ in parabolic orbits, a significant fraction of the resulted HVSs have velocities larger than the detected values. They also study the interactions between the $\mathrm{BH}$ and binary stars bound to it. In this case, the binary would experience multiple encounters with the BH. Due to the cumulative tidal effect, the probability that the binary will be broken up becomes substantial even at a large penetration factor $D$. The observed distribution would be reproduced if binary stars slowly diffuse onto low angular momentum orbits and most of them are broken up at a large 
distance with small ejection velocities. This could provide an alternative interesting solution to the problem.

In conclusion, data at this stage seem to suggest the need for more power to wider binaries to account for the paucity of high-velocity stars. The above discussion is, of course, not conclusive or exhaustive, given the quality and quantity of the data. Nevertheless, it shows the great potentiality of HVSs to constrain physical properties of the Galaxy and the Galactic Bulge, once a larger data set of HVSs is collected.

\section{DISCUSSION AND CONCLUSIONS}

In this paper, we derive the velocity distribution in the Galactic halo of stars ejected from a stellar binary system, following a tidal interaction with the supermassive $\mathrm{BH}$ at the center of our Galaxy.

The magnitude of the ejection velocity, after the star has climbed out of the BH potential well, was calculated by SKR and Kobayashi et al. (2012). In this paper, we assume a statistical description of the binaries injected into the tidal sphere of the $\mathrm{BH}$ (mass and separation distributions) and we consider two limiting cases for the probability that binaries are tidally disrupted: one that is linearly proportional to the binary separation (full losscone regime) and one in which all binaries are disrupted with equal probability (empty loss cone). In the former case, looser binaries are disrupted more easily, and since they give rise to lower ejection velocities, the velocity distributions in this regime are generally steeper than in the empty loss cone. A mixed case can be straightforwardly derived from our results, and we use it in our final discussion. Finally, we account for the deceleration due to the Galactic potential and the finite lifetime of stars in order to obtain the star velocity distribution as they travels in the Galactic halo.

Some ingredients may be treated less crudely once the quality of data improve and require more sophisticated models. An improvement could be to use a detailed model for the Galaxy potential, which includes different Galactic components (disk, bulge, and halo). It can be a spherically symmetric model (Kenyon et al. 2008) or one that allows for a degree of triaxiality in the halo (Gnedin et al. 2005). A larger HVS data sample - spread over many scales - may in principle map the shape and depth of the Galactic potential (Gnedin et al. 2005; Yu \& Madau 2007).

As discussed in Section 5, one should in principle take a star age distribution, as stars may be injected in the tidal sphere of the $\mathrm{BH}$ at different times in their life. This becomes important when the star travel time to a given Galactocentric distance becomes comparable to the star lifetime, which occurs for stars more massive than currently observed. Finally, the highest velocity tail ( $\gg$ a few $1000 \mathrm{~km} \mathrm{~s}^{-1}$ ), which is given by contact binaries, may be depleted by star tidal disruptions, because the tidal radius for the more massive star of the binary is comparable to the binary tidal radius. Currently, these fastest speeds are not observed and therefore we have not included in this paper such modifications.

Regardless of the above caveats, our results show that, for a given HVS mass, the velocity distributions at large Galactic distances have a steep rise up to the peak, which depends only on the Galactic deceleration model. In our model, where we assume that the stars are far enough that all the deceleration has already taken place, the peak velocity is related to the escape velocity from the Galaxy. For velocities larger than the peak, the distribution eventually starts to decrease. The slope following the peak depends on the binary separation distribution, while the very highest velocity branch bears imprint of the binary mass distribution.

In this paper, we make a first attempt to compare our model with the current data (Section 6.3). The observed velocity range is quite narrow $\left(380-720 \mathrm{~km} \mathrm{~s}^{-1}\right)$ and there are no detentions of HVSs beyond $720 \mathrm{~km} \mathrm{~s}^{-1}$, though there is no obvious observational bias that explains it. By considering cumulative velocity distributions (Figure 7), we find a significant discrepancy between the observational data and theoretical distributions based on conventional assumptions (see also Zhang et al. 2010). The detected HVSs are much more concentrated in low velocities around $300-400 \mathrm{~km} \mathrm{~s}^{-1}$. To explain the data, we need velocity distributions with an index $\beta \sim 8$ much steeper than our fiducial values: $\beta=0$ (empty loss cone) and 2 (full loss cone). Note that in the empty loss-cone regime which theoretical arguments favor, the discrepancy is even larger. If the distribution has a sharp cut-off at $v \sim 800 \mathrm{~km} \mathrm{~s}^{-1}$ (e.g., due to a larger minimum separation $a_{\min } \sim 10 R_{\odot}$ ), a shallower distribution $(\beta \sim 3)$ would be consistent with the data. These might indicate that the binary separation distribution in the Galactic bulge is not flat in logarithmic space as observed in more local massive binaries, but has more power toward larger separations, enhancing smaller velocities (another interesting possibility is the multiple encounter model studied by Zhang et al. 2010, 2013). A possible cut-off at $\sim 10 R_{\odot}$ might be related to the binary formation/evolution process or to the injection mechanism which could filter out tight binaries.

At this stage, our model comparison with data is just qualitative, as we need to wait for future missions, like the Gaia satellite, ${ }^{9}$ to detect a few hundreds of HVSs in a wider range of masses. Nevertheless, this comparison-even in its limitation-shows the great potential of our modeling to extract information on the bulge structure and stellar content, and on the Galaxy structure in general.

\section{REFERENCES}

Antonini, F., Faber, J., Gualandris, A., \& Merritt, D. 2010, ApJ, 713, 90 Bromley, B. C., Kenyon, S. J., Geller, M. J., et al. 2006, ApJ, 653, 1194 Brown, W. R., Anderson, J., Gnedin, O. Y., et al. 2010, ApJ, 719, 23 Brown, W. R., Cohen, J. G., Geller, M. J., \& Kenyon, S. J. 2012a, ApJ, 754, 2 Brown, W. R., Geller, M. J., \& Kenyon, S. J. 2009a, ApJ, 690, 1639 Brown, W. R., Geller, M. J., \& Kenyon, S. J. 2012b, ApJ, 751, 55

Brown, W. R., Geller, M. J., Kenyon, S. J., \& Bromley, B. C. 2009b, ApJL, 690, L69

Brown, W. R., Geller, M. J., Kenyon, S. J., \& Kurtz, M. J. 2005, ApJL, 622, L33

Brown, W. R., Geller, M. J., Kenyon, S. J., Kurtz, M. J., \& Bromley, B. C. 2007, ApJ, 671, 1708

Duquennoy, A., \& Mayor, M. 1991, A\&A, 248, 485

Edelmann, H., Napiwotzki, R., Heber, U., Christlieb, N., \& Reimers, D. 2005, ApJL, 634, L181

Eggleton, P. P. 1983, ApJ, 268, 368

Genzel, R., Thatte, N., Krabbe, A., Kroker, H., \& Tacconi-Garman, L. E. 1996, ApJ, 472, 153

Ghez, A. M., Klein, B. L., Morris, M., \& Becklin, E. E. 1998, ApJ, 509, 678

Ghez, A. M., Salim, S., Weinberg, N. N., et al. 2008, ApJ, 689, 1044

Ginsburg, I., \& Loeb, A. 2006, MNRAS, 368, 221

Gnedin, O. Y., Gould, A., Miralda-Escud, J., \& Zentner, A. R. 2005, ApJ, 634,344

Gualandris, A., Portegies Zwart, S., \& Sipior, M. S. 2005, MNRAS, 363, 223

Hansen, C. J., Kawaler, S. D., \& Trimble, V. 1994, Stellar Interiors: Physical Principles, Structure, and Evolution (New York: Springer)

Hansen, C. J., Kawaler, S. D., \& Trimble, V. 2004, Stellar Interiors: Physical Principles, Structure, and Evolution (New York: Springer)

Hills, J. G. 1988, Natur, 331, 687

Hirsch, H. A., Heber, U., O’Toole, S. J., \& Bresolin, F. 2005, A\&A, 444, L61

\footnotetext{
9 The Gaia mission blasted off in 2013 December.
} 
Kenyon, S. J., Bromley, B. C., Geller, M. J., \& Brown, W. R. 2008, ApJ, 680,312

Kiminki, D. C., \& Kobulnicky, H. A. L. 2012, ApJ, 751, 4

Kobayashi, S., Hainick, Y., Sari, R., \& Rossi, E. M. 2012, ApJ, 748, 105

Kobulnicky, H. A., \& Fryer, C. L. 2007, ApJ, 670, 747

Kollmeier, J. A., Gould, A., Knapp, G., \& Beers, T. C. 2009, ApJ, 697,1543

Kollmeier, J. A., Gould, A., Rockosi, C., et al. 2010, ApJ, 723, 812

Krumholz, M. R., Klein, R. I., McKee, C. F., Offner, S. S. R., \& Cunningham, A. J. 2009, Sci, 323, 754

Levin, Y., \& Beloborodov, A. M. 2003, ApJ, 590, 33

Lightman, A. P., \& Shapiro, S. L. 1977, ApJ, 211, 244

Lu, J. R., Ghez, A. M., Hornstein, S. D., et al. 2009, ApJ, 690, 1463
Lu, Y., Zhang, F., \& Yu, Q. 2010, ApJ, 709, 1356

Perets, H. B., \& Gualandris, A. 2010, ApJ, 719, 220

Perets, H. B., Hopman, C., \& Alexander, T. 2007, ApJ, 656, 709

Sari, R., Kobayashi, S., \& Rossi, E. M. 2010, ApJ, 708, 605

Sesana, A., Haardt, F., \& Madau, P. 2007, MNRAS, 379, L45

Schödel, R., Ott, T., Genzel, R., et al. 2003, ApJ, 596, 1015

Smith, M. C., Ruchti, G. R., Helmi, A., et al. 2007, MNRAS, 379, 755

Vanbeveren, D., De Donder, E., Van Bever, J., Van Rensbergen, W., \& De Loore, C. 1998, NewA, 3, 443

Yu, Q., \& Madau, P. 2007, ApJ, 379, 1293

Yu, Q., \& Tremaine, S. 2003, ApJ, 599, 1129

Zhang, F., Lu, Y., \& Yu, Q. 2010, ApJ, 722, 1744

Zhang, F., Lu, Y., \& Yu, Q. 2013, ApJ, 768, 153 